\title{
Thirty-day mortality after percutaneous gastrostomy by endoscopic versus radiologic placement: a systematic review and meta-analysis
}

\author{
Joo Hyun Lim*, Seung Ho Choi*, Changhyun Lee, Ji Yeon Seo, Hae Yeon Kang, Jong In Yang, Su Jin Chung, \\ Joo Sung Kim \\ Department of Internal Medicine and Healthcare Research Institute, Healthcare System Gangnam Center, Seoul National University Hospital, \\ Seoul, Korea
}

Background/Aims: A percutaneous gastrostomy can be placed either endoscopically (percutaneous endoscopic gastrostomy, PEG) or radiologically (radiologically-inserted gastrostomy, RIG). However, there is no consistent evidence of the safety and efficacy of PEG compared to RIG. Recently, 30-day mortality has become considered as the most important surrogate index for evaluating the safety and efficacy of percutaneous gastrostomy. The aim of this meta-analysis was to compare the 30day mortality rates between PEG and RIG. Methods: Major electronic databases (MEDLINE, Embase, Scopus, and Cochrane library) were queried for comparative studies on the two insertion techniques of gastrostomy among adults with swallowing disturbance. The primary outcome was the 30-day mortality rate after gastrostomy insertion. Forest and funnel plots were generated for outcomes using STATA version 14.0. Results: Fifteen studies $(\mathrm{n}=2,183)$ met the inclusion criteria. PEG was associated with a lower risk of 30-day mortality after tube placement compared with RIG (odds ratio, 0.60; 95\% confidence interval $[\mathrm{CI}], 0.38-0.94 ; P=0.026$ ). The pooled prevalence of 30-day mortality of PEG was $5.5 \%$ (95\% CI, $4.0 \%-6.9 \%$ ) and that of RIG was $10.5 \%$ (95\% CI, 6.8\%-14.3\%). No publication bias was noted. Conclusions: The present meta-analysis demonstrated that PEG is associated with a lower probability of 30-day mortality compared to RIG, suggesting that PEG should be considered as the first choice for long-term enteral tube feeding. Further prospective randomized studies are needed to evaluate and compare the safety of these two different methods of gastrostomy. (Intest Res 2016;14:333-342)

Key Words: Gastrostomy; Endoscopy; Fluoroscopy; Mortality

\section{INTRODUCTION}

Gastrostomy feeding is recommended for patients with dysphagia to improve nutrition. ${ }^{1}$ Previously, gastrostomy tubes were placed solely using the open surgical technique.

Received December 18, 2015. Revised February 10, 2016

Accepted February 22, 2016.

Correspondence to Changhyun Lee, Department of Internal Medicine and

Healthcare Research Institute, Healthcare System Gangnam Center, Seoul

National University Hospital, 152 Teheran-ro, Gangnam-gu, Seoul 06236,

Korea. Tel: +82-2-2112-5578, Fax: +82-2-2112-5635, E-mail: mdchlee@

gmail.com

*These authors contributed equally to this study.

Financial support: None. Conflict of interest: None.
However, percutaneous endoscopic gastrostomy (PEG) has replaced surgical placement since it was introduced by Gauderer and Ponsky in $1980 .^{2}$ Radiologically-inserted gastrostomy (RIG) was also introduced in 1981 and has been used as an alternative to PEG for enteral feeding with a high rate of technical success under local anesthesia. ${ }^{3}$ Although PEG and RIG are both considered minimally invasive, they are associated with considerable early mortality (30-day mortality), which has been reported as $5.8 \%$ to $28 \%$ for PEG and $1 \%$ to $40 \%$ for RIG. ${ }^{4-9}$ Furthermore, various complications such as wound infections, hemorrhage, hematoma formation, aspiration pneumonia, or gastric perforation may develop after gastrostomy. Although pneumoperitoneum is

\footnotetext{
(c) Copyright 2016. Korean Association for the Study of Intestinal Diseases. All rights reserved.

This is an Open Access article distributed under the terms of the Creative Commons Attribution Non-Commercial License (http://creativecommons.org/licenses/by-nc/4.0)

which permits unrestricted non-commercial use, distribution, and reproduction in any medium, provided the original work is properly cited.
} 
a complication which is usually considered minor, a previous study reported mortality related to pneumoperitoneum after PEG insertion. ${ }^{10}$ Considering that less invasive alternatives do exist, such as a nasogastric tube insertion for shortterm tube feeding, it is important to evaluate the safety of gastrostomy. Thirty-day mortality is one of the most important surrogate indices for evaluating the safety of percutaneous gastrostomy. ${ }^{1,11,12}$ However, no systematic reviews or meta-analyses have compared PEG and RIG in terms of 30day mortality rates. In the absence of sufficient evidence, the choice between PEG and RIG is largely based on expert opinions or institutional preference. The aim of the present study was to perform a systemic review and meta-analysis comparing the 30-day mortality rates after PEG and RIG.

\section{METHODS}

The protocol for this review was developed prospectively. The checklist and flow chart of the preferred reporting items for systematic review and meta-analyses (PRISMA) statement were used for the review process and data extraction. ${ }^{13}$ We prepared a prespecified protocol of the specific patients (patients with dysphagia), interventions (PEG), controls (RIG), the primary outcome (the 30-day mortality), the criteria for study selection, and the assessment of study quality.

\section{Search Strategy}

To identify all available studies, a detailed search was conducted according to the PRISMA guidelines. ${ }^{12}$ A systematic search was performed in major electronic databases (MEDLINE, Cochrane library, Scopus, and Embase) using the following search terms: "Gastrostomy" AND "Endoscopic" AND "Radiologic*". The search was restricted to literature from January 1980 to May 2015. Non-English or non-Korean language trials were translated and included.

\section{Study Selection}

The following selection criteria were applied: (1) patients with dysphagia or in need of prolonged tube feeding; (2) randomized controlled trials (RCT) or other comparative studies comparing 30-day mortality between PEG and RIG; (3) outcome measures of 30-day mortality; and (4) studies that were published in peer-reviewed journals. As there have been only two randomized control trials comparing PEG versus RIG to date, this analysis included both prospective and retrospective comparative studies.
Studies were excluded when they met any of the following criteria: (1) preclinical studies, case reports, case series, or review articles; (2) studies with RIG placement after failure of PEG; (3) studies with no data pertaining to 30-day mortality rates; or (4) studies with no cases of 30-day mortality in either the PEG and the RIG group or duplicated reports from the same patient.

The results of the database search were imported into EndNote X7 software (Thomson Reuters, Philadelphia, PA, USA) and combined into one group to remove duplicated references. Two authors (J.H.L. and S.H.C.) independently assessed the titles and abstracts of all the studies identified by the search. Full texts of potentially eligible articles were obtained and reviewed to assess whether the studies fulfilled the inclusion criteria. Any disagreements between reviewers were resolved through discussion. When an agreement was not achieved, another author (C.L.) was consulted.

\section{Quality Assessment}

The quality of the RCTs was assessed using the Jadad scale. ${ }^{14}$ The quality and risk of bias of nonrandomized comparative studies (NRCT) was assessed using the methodological index for nonrandomized studies (MINORS). ${ }^{15}$

\section{Data Extraction}

Two independent authors (J.H.L. and S.H.C.) analyzed each article and extracted data using a predefined data extraction worksheet. The following information was extracted from each article: title, first author, year of publication, country of origin, study design, patient demographics, the interventions being compared, number of patients, and the 30day mortality rate.

\section{Statistical Analysis and Publication Bias Assessment}

A meta-analysis was carried out using STATA version 14 (Stata Corp, Texas, TX, USA). We used the STATA metan command for fixed-model (Mantel-Haenszel) analysis and random-effect (DerSimonian and Laird) analysis. We excluded trials with no cases of 30-day mortality in either intervention groups. Differences between the PEG and RIG groups were expressed as the pooled OR with $95 \%$ CI. We also used the STATA metan command to create a forest plot and calculate the pooled prevalence. Publication bias was presented graphically by funnel plots of the standard difference in means versus the standard error using the "metafun- 

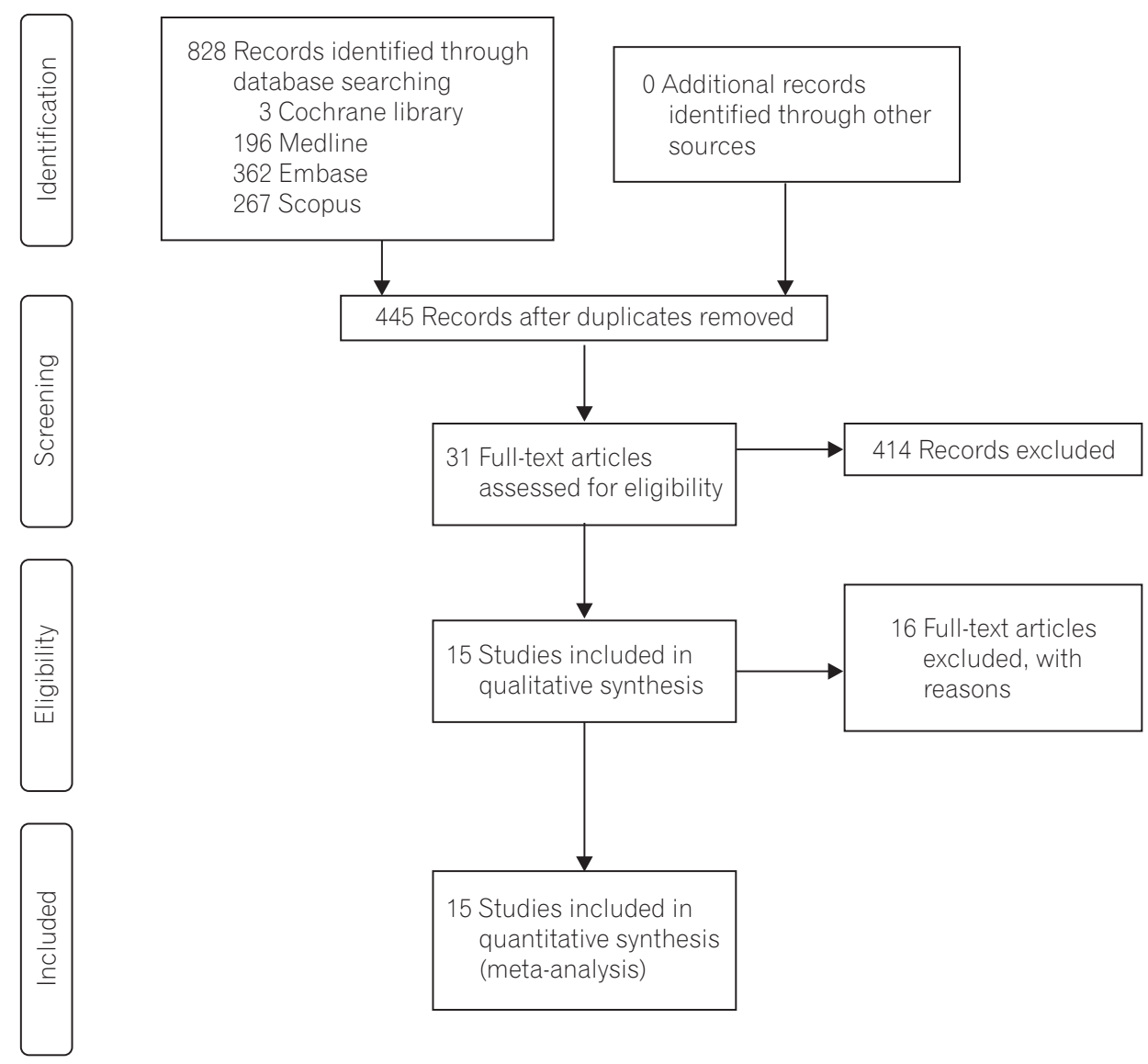

Fig. 1. Preferred reporting items for systematic review and meta-analyses diagram of the included articles.

Table 1. Characteristics of Trials Included in the Meta-Analysis

\begin{tabular}{|c|c|c|c|c|c|c|}
\hline \multirow{2}{*}{ Author (year) } & \multirow{2}{*}{ Country } & \multirow{2}{*}{ Study type } & \multirow{2}{*}{$\begin{array}{l}\text { Quality } \\
\text { score }^{a}\end{array}$} & \multirow{2}{*}{$\begin{array}{l}\text { Underlying } \\
\text { disease }\end{array}$} & \multicolumn{2}{|c|}{ Sample size } \\
\hline & & & & & PEG & $\overline{\text { RIG }}$ \\
\hline Lewis et al. $(2014)^{16}$ & UK & Randomized controlled trial & $3 / 5$ & Mixed & 34 & 31 \\
\hline ProGas Study Group (2015) ${ }^{9}$ & UK & Prospective & $22 / 24$ & MND & 157 & 114 \\
\hline Laskaratos et al. $(2013)^{8}$ & UK & Retrospective & $20 / 24$ & Mixed & 53 & 40 \\
\hline La Nauze et al. $(2012)^{20}$ & Australia & Retrospective & $18 / 24$ & Mixed & 80 & 97 \\
\hline Rio et al. $(2010)^{22}$ & UK & Retrospective & $17 / 24$ & MND & 21 & 121 \\
\hline Leeds et al. $(2010)^{23}$ & UK & Prospective & $22 / 24$ & Mixed & 233 & 170 \\
\hline Blondet et al. $(2010)^{24}$ & France & Retrospective & $18 / 24$ & MND & 21 & 22 \\
\hline Desport et al. $(2005)^{32}$ & France & Prospective & $19 / 24$ & MND & 30 & 20 \\
\hline Neeff et al. $(2003)^{33}$ & New Zealand & Retrospective & $17 / 24$ & HEN & 56 & 18 \\
\hline Wollman and D'Agostino $(1997)^{42}$ & USA & Retrospective & $20 / 24$ & Mixed & 79 & 46 \\
\hline
\end{tabular}

a Jadad score for randomized controlled trials, methodological index for nonrandomized studies (MINORS) score for nonrandomized comparative studies. PEG, percutaneous endoscopic gastrostomy; RIG, radiologically-inserted gastrostomy; MND, motor neuron disease; HEtN, head and neck cancer. 
Table 2. Indications for Gastrostomy

\begin{tabular}{|c|c|c|c|c|c|c|c|c|}
\hline \multirow{2}{*}{ Author } & \multicolumn{2}{|c|}{ HEN } & \multicolumn{2}{|c|}{ Neurological impairment $^{\mathrm{a}}$} & \multicolumn{2}{|c|}{ Others } & \multicolumn{2}{|c|}{ Total } \\
\hline & PEG & RIG & PEG & RIG & PEG & RIG & PEG & RIG \\
\hline Lewis et al. ${ }^{16}$ & 4 & 7 & 20 & 20 & 4 & 1 & 34 & 31 \\
\hline Hoffer et al. ${ }^{39}$ & 7 & 9 & 54 & 55 & 8 & 2 & 69 & 66 \\
\hline ProGas Study Group $^{9}$ & 0 & 0 & 157 & 114 & 0 & 0 & 157 & 114 \\
\hline Laskaratos et al. ${ }^{8}$ & 8 & 9 & 34 & 21 & 11 & 10 & 53 & 40 \\
\hline Allen et al. ${ }^{19}$ & 0 & 0 & 57 & 51 & 0 & 0 & 57 & 51 \\
\hline La Nauze et al..$^{20}$ & 16 & 31 & 48 & 54 & 15 & 16 & 80 & 97 \\
\hline Rio et al. $^{22}$ & 0 & 0 & 21 & 121 & 0 & 0 & 21 & 121 \\
\hline Leeds et al. ${ }^{23}$ & 69 & 106 & 111 & 46 & 53 & 18 & 233 & 170 \\
\hline Blondet et al. ${ }^{24}$ & 0 & 0 & 21 & 22 & 0 & 0 & 21 & 22 \\
\hline Galaski et al. $^{26}$ & Unknown & Unknown & Unknown & Unknown & Unknown & Unknown & 30 & 44 \\
\hline Eze et al. ${ }^{28}$ & 44 & 15 & 0 & 0 & 0 & 0 & 44 & 15 \\
\hline Silas et al..$^{31}$ & 41 & 134 & 89 & 37 & 47 & 22 & 177 & 193 \\
\hline Desport et al. ${ }^{32}$ & 0 & 0 & 30 & 20 & 0 & 0 & 30 & 20 \\
\hline Neeff et al. ${ }^{33}$ & 56 & 18 & 0 & 0 & 0 & 0 & 56 & 18 \\
\hline Wollman and D'Agostino ${ }^{42}$ & 13 & 25 & 84 & 25 & 17 & 18 & 79 & 46 \\
\hline
\end{tabular}

${ }^{a}$ Neurological impairment includes motor neuron disease, cerebrovascular disease, cerebral tumor, and cerebral trauma.

HEN, head and neck cancer; PEG, percutaneous endoscopic gastrostomy; RIG, radiologically-inserted gastrostomy.

Table 3. Thirty-Day Mortality Rate

\begin{tabular}{|c|c|c|}
\hline \multirow{2}{*}{ Author } & \multicolumn{2}{|c|}{ 30-Day mortality } \\
\hline & PEG & RIG \\
\hline Lewis et al. ${ }^{16}$ & $2 / 34$ & $2 / 31$ \\
\hline Hoffer et al. ${ }^{39}$ & $5 / 69$ & $4 / 66$ \\
\hline ProGas Study Group ${ }^{9}$ & $5 / 163$ & $4 / 121$ \\
\hline Laskaratos et al. $^{8}$ & $6 / 53$ & $14 / 40$ \\
\hline Allen et al..$^{19}$ & $4 / 57$ & $6 / 51$ \\
\hline La Nauze et al. ${ }^{20}$ & $3 / 80$ & $5 / 97$ \\
\hline Rio et al. ${ }^{22}$ & $2 / 21$ & $12 / 121$ \\
\hline Leeds et al. ${ }^{23}$ & $25 / 233$ & $26 / 170$ \\
\hline Blondet et al. ${ }^{24}$ & $2 / 21$ & $2 / 22$ \\
\hline Galaski et al. ${ }^{26}$ & $1 / 30$ & $8 / 44$ \\
\hline Eze et al. ${ }^{28}$ & $1 / 44$ & $5 / 15$ \\
\hline Silas et al..$^{31}$ & $8 / 177$ & $6 / 193$ \\
\hline Desport et al. ${ }^{32}$ & $4 / 30$ & $2 / 20$ \\
\hline Neeff et al. ${ }^{33}$ & $3 / 56$ & $6 / 18$ \\
\hline Wollman and D'Agostino ${ }^{42}$ & $8 / 79$ & $4 / 46$ \\
\hline
\end{tabular}

PEG, percutaneous endoscopic gastrostomy; RIG, radiologicallyinserted gastrostomy. nel" command and assessed by the Egger's test using the "metabias" command. Subgroup analysis was performed in each group of patients with motor neuron disease and head and neck cancer.

\section{RESULTS}

\section{Search Results}

According to the PRISMA flowchart, the selection results are reported in Fig. $1 .{ }^{13}$ We identified 828 citations through the electronic database search, and 445 citations remained after duplicates were removed. Initial screening of the titles and abstracts resulted in the exclusion of 414 citations, and full texts of the remaining 31 citations were reviewed in detail. ${ }^{8,9,16-44}$ An additional 16 citations were excluded after reviewing the full articles, for the following reasons: duplicated reports, ${ }^{42-44}$ noncomparative studies, ${ }^{37}$ studies with RIG placement after failure of $\mathrm{PEG}^{25,35}$ no data present on 30-day mortality, ${ }^{29,40,41}$ no reported case of 30-day mortality, ${ }^{19,27,30,34,36,38}$ and review articles. ${ }^{17,21}$ Fifteen published studies including two RCTs ${ }^{16,39}$ and $13 \mathrm{NRCTs}^{8,9,19,20,22-24,26,28,31-33,42}$ were included in the final analysis. 


\section{Baseline Characteristics of the Included Studies}

A total of 1,135 patients with PEG insertion and 1,048 patients with RIG insertion were included in the 15 studies.
Blinding was not applied to the two RCTs, and the Jadad scores of the two studies were 2 and 3 . The quality of the NRCTs was measured by the MINORS score, and the estimated mean MINORS score was 18.6 (95\% CI, 17.27-19.95).

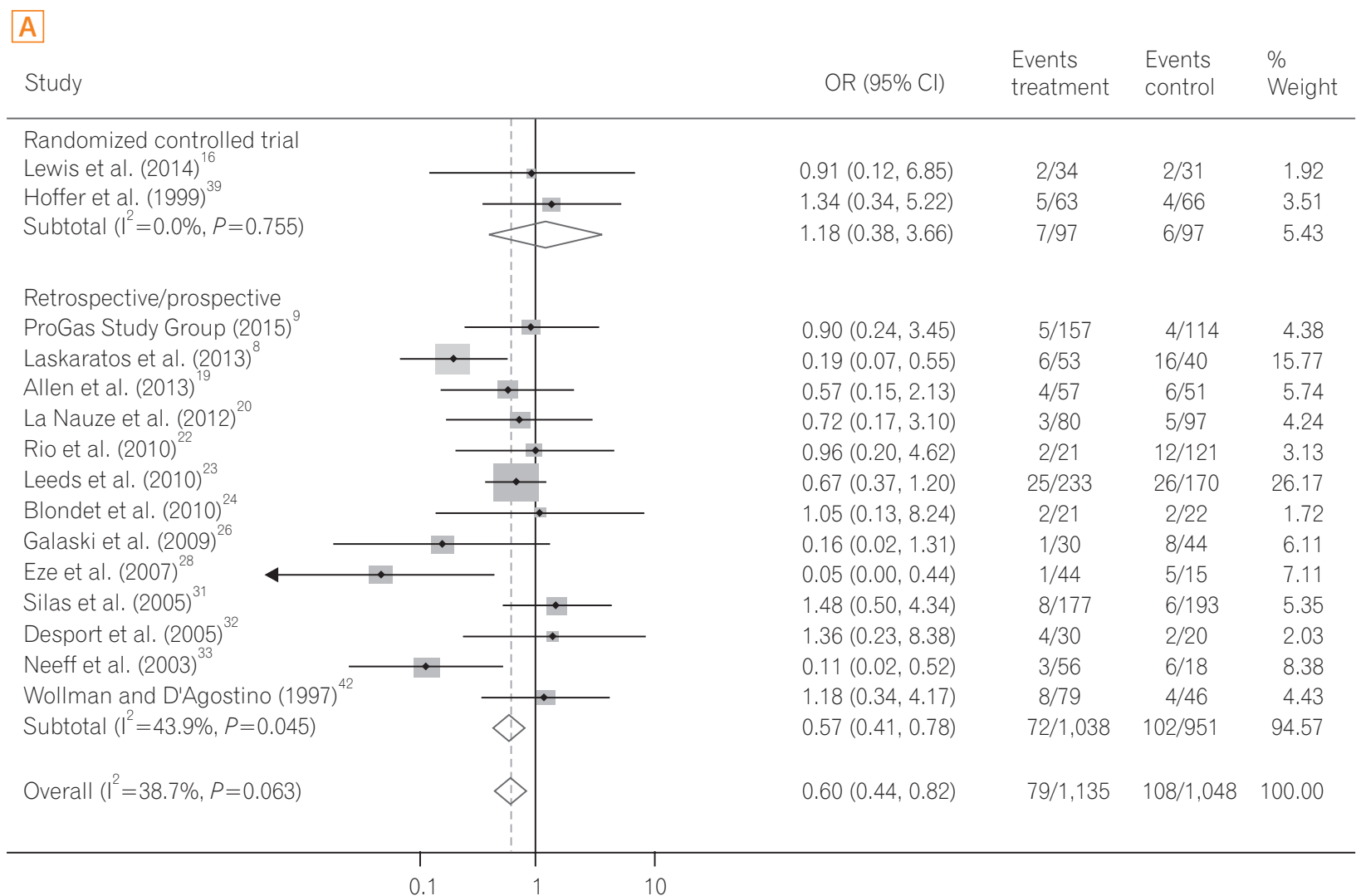

\section{B}

Study

ID

OR $(95 \% \mathrm{Cl})$

$\%$

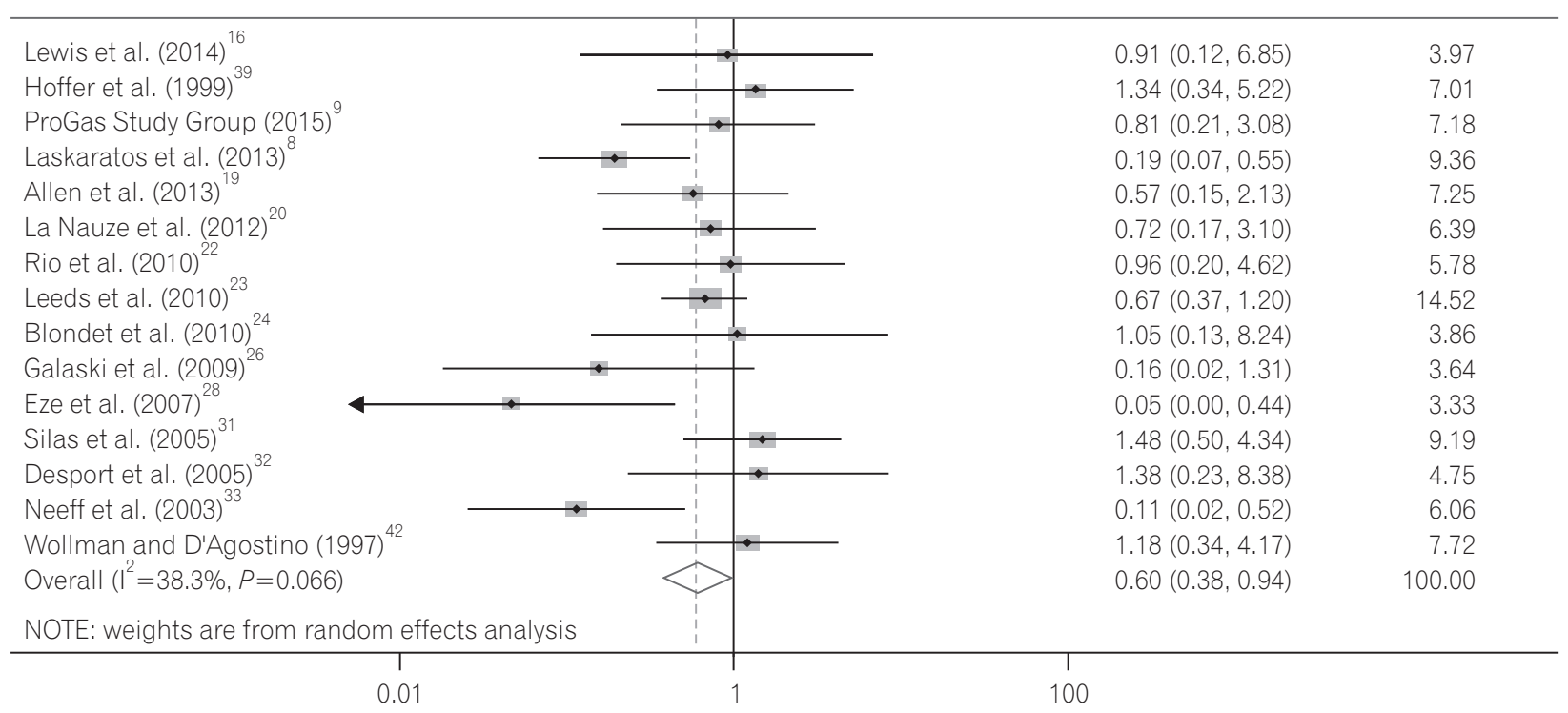

Fig. 2. Forest plot of 30-day mortality after gastrostomy tube insertion. (A) Fixed effect model. (B) Random effect model. 
The characteristics of these studies and quality assessment scores are detailed in Table 1 . These studies were published from 1997 to 2015. All of the studies were performed in Western countries ( six in the UK, four in the USA, two in

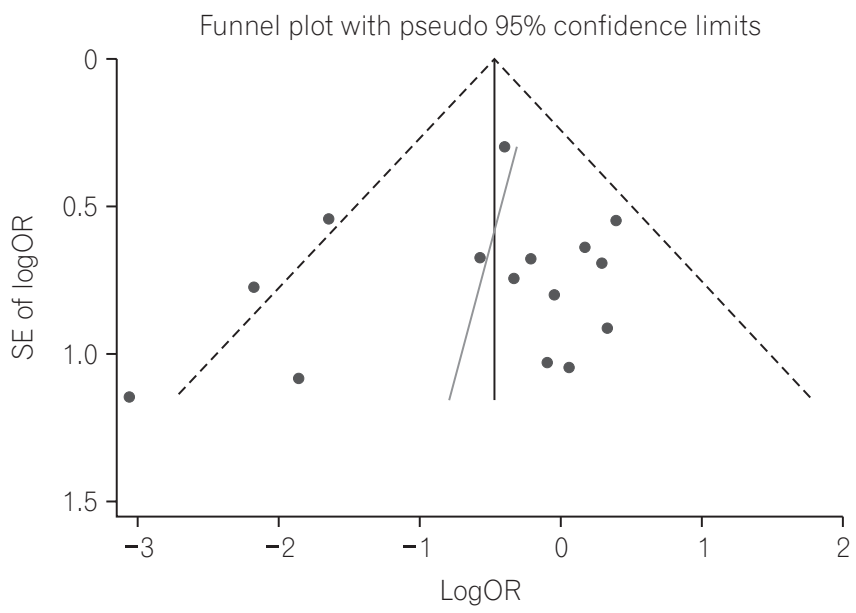

Fig. 3. Funnel plot of 30-day mortality after gastrostomy tube insertion.
France, and one in Australia, Canada, and New Zealand, each). The underlying diseases of the patients varied (mixed in eight studies, motor neuron disease in five studies, head and neck cancer in two studies), and the sample sizes of the studies ranged from 43 to 403 patients. Detailed indications are presented in Table 2.

\section{The 30-Day Mortality Rate Following PEG versus RIG}

The 30-day mortality rates reported by each study are shown in Table 3 and Fig. 2A. A pooled analysis of the two RCTs with the fixed model showed no difference between the two groups (OR, 1.18; 95\% CI, $0.38-3.66 ; P=0.770)$. As this result was thought to be due to the small number of RCTs included, we performed a pooled analysis with RCTs and NRCTs combined. The pooled analysis of the ORs demonstrated a statistically significant reduction of 30-day mortality rates in the PEG groups compared with the RIG groups (OR, 0.60; 95\% CI, 0.44-0.82; $P=0.001$ ) (Fig. 2A) in a fixed effects model with moderate heterogeneity (heterogeneity $\left.\chi^{2}=22.68, \mathrm{I}^{2}=38.7 \%, P=0.063\right)$. Because of this moderate het

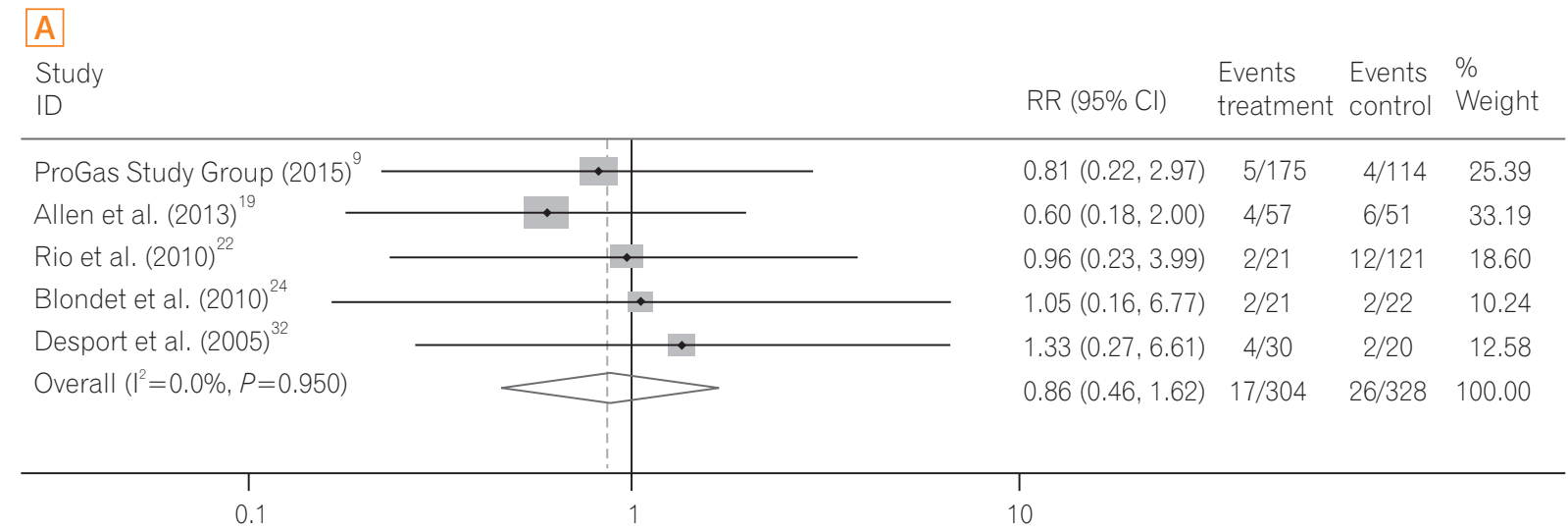

B

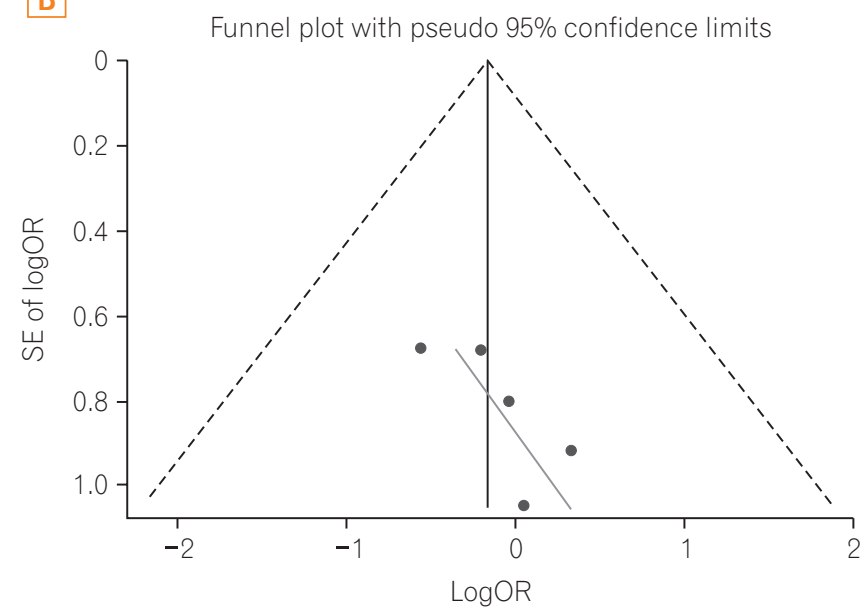

Fig. 4. Subgroup analysis of patients with motor neuron disease. $(A)$ Forest plot of 30-day mortality after gastrostomy tube insertion. (B) Funnel plot of 30-day mortality after gastrostomy tube insertion. 
erogeneity, we next used a random effects model to perform a pooled analysis of the ORs. In the random effects model, the pooled analysis of the ORs also demonstrated a statistically significant reduction of 30-day mortality rates in the PEG groups (OR, 0.60; 95\% CI, 0.38-0.94; $P=0.026$ ) (Fig. 2B). The funnel plot assay (Fig. 3$)$ and the Egger's test $(P=0.542)$ did not show evidence of publication bias.

Next, we calculated the pooled 30-day mortality rate in the PEG and RIG groups. The pooled prevalence of 30-day mortality of the PEG group was $5.5 \%(95 \% \mathrm{CI}, 4.0 \%-6.9 \%)$ in a fixed effects model with low heterogeneity (heterogeneity $\left.\chi^{2}=17.09, \mathrm{I}^{2}=18.1 \%, P=0.251\right)$. The pooled prevalence of 30 day mortality of the RIG group was $6.7 \%$ (95\% CI, 5.1\%-8.3\%) in a fixed effects model with high heterogeneity (heterogeneity $\left.\chi^{2}=50.92, \mathrm{I}^{2}=72.5 \%, P<0.001\right)$. Because of this high heterogeneity, we then applied a random effects model. In the random effects model, the pooled prevalence of 30-day mortality of the RIG group was $10.5 \%$ (95\% CI, $6.8 \%-14.3 \%)$.

\section{The 30-Day Mortality Rate Following PEG versus RIG in Patients with Motor Neuron Disease}

A total of five studies ${ }^{9,19,22,24,32}$ were performed in patients with motor neuron disease. Pooled analysis of the ORs showed no significant difference in 30-day mortality between the PEG group and RIG group (OR, 0.84; 95\% CI, $0.43-1.69 ; P=0.637$ ) (Fig. 4A) with low heterogeneity (heterogeneity $\chi^{2}=0.71, \mathrm{I}^{2}<0.1 \%, P=0.950$ ). The funnel plot assay (Fig. $4 \mathrm{~B})$ and the Egger's test $(P=0.124)$ did not show evidence of publication bias.

\section{The 30-Day Mortality Rate Following PEG versus RIG in Patients with Head and Neck Cancer}

A total of three studies ${ }^{23,28,33}$ were performed in patients with head and neck cancer including a subgroup analysis study. ${ }^{23}$ The pooled OR of 30-day mortality was significantly reduced in the PEG group compared to the RIG group (OR, 0.09; 95\% CI, 0.03-0.28; $P<0.001$ ) (Fig. 5A) with low heterogeneity (heterogeneity $\chi^{2}=0.51, \mathrm{I}^{2}<0.1 \%, P=0.775$ ). The funnel plot assay (Fig. 5B) and the Egger's test $(P=0.060)$ did not show evidence of publication bias.

\section{DISCUSSION}

This is the first systematic review and meta-analysis com-

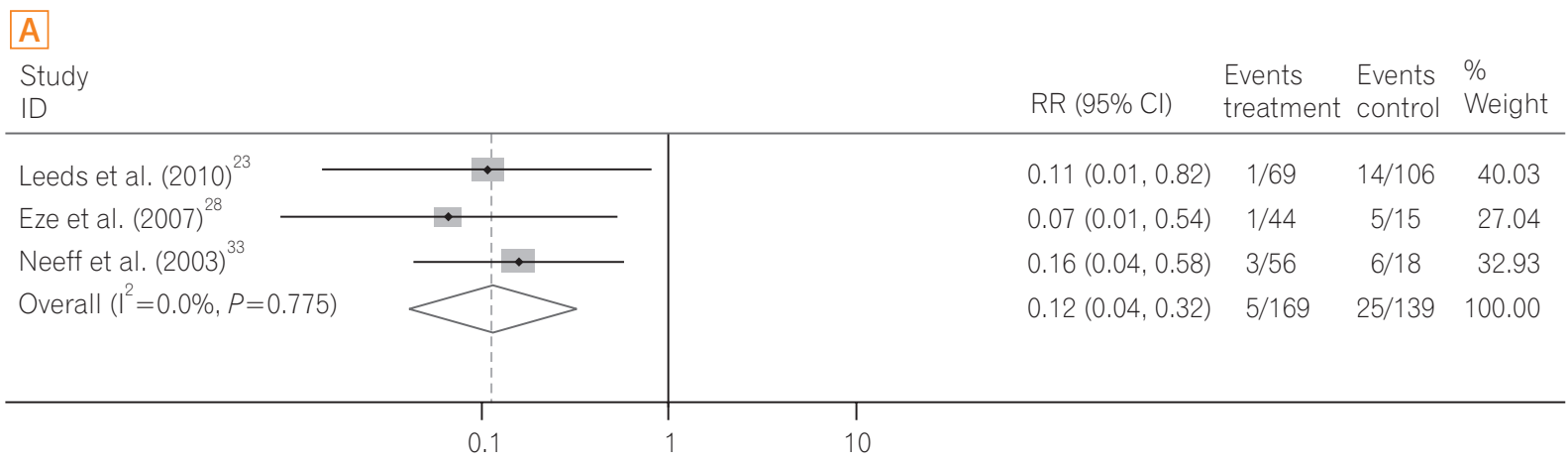

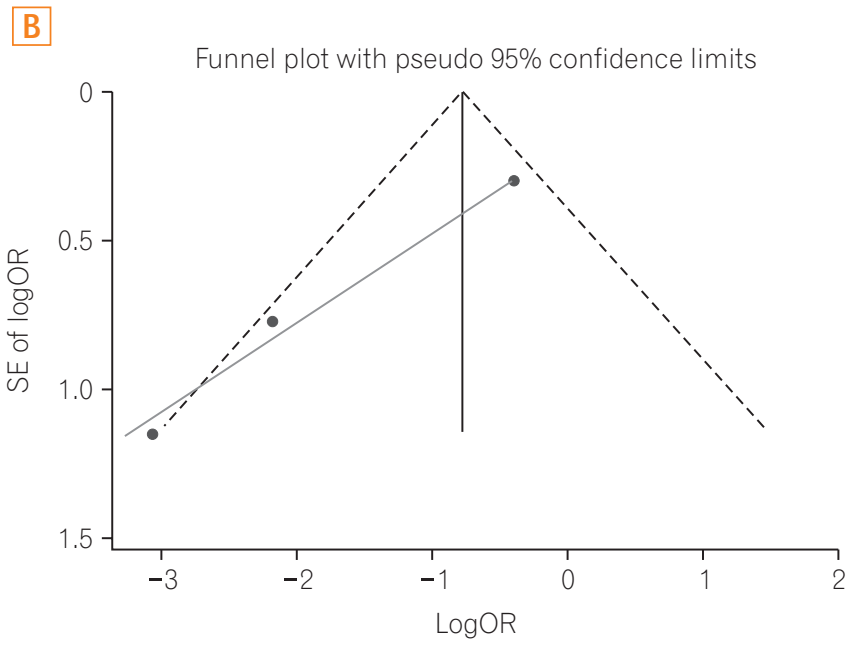

Fig. 5. Subgroup analysis in patients with head and neck cancer. (A) Forest plot of 30-day mortality after gastrostomy tube insertion. (B) Funnel plot of 30-day mortality after gastrostomy tube insertion. 
paring the 30-day mortality rates of PEG versus RIG. We reviewed 30-day mortality after PEG or RIG by summarizing 15 peer-reviewed studies, including 1,135 patients who underwent PEG and 1,048 patients who underwent RIG. A significantly lower risk of 30-day morality was found in the PEG group compared to that in the RIG group (OR, 0.60; 95\% CI, $0.38-0.94 ; P=0.026)$. This finding supports the current trend of clinical practice, in which PEG is the method of choice for long-term enteral tube feeding, and RIG is an alternative method with a high rate of technical success for those who refuse endoscopy or are unsuitable for PEG.

PEG and RIG placement are generally known as safe and feasible procedures with low mortality rates. ${ }^{9}$ In this metaanalysis, the pooled 30-day mortality rate of the PEG group was $5.5 \%$ (95\% 4.0\%-6.9\%) and that of the RIG group was $10.5 \%$ (95\% CI, 6.8\%-14.3\%). These findings suggest that more careful exploration of the risks and benefits of the procedure is needed, especially for RIG, because less invasive methods exist for short-term tube feeding, such as nasogastric tube insertion.

The reason for the lower 30-day mortality rate in the PEG group could be due to the following: first, administration of prophylactic antibiotics is recommended in PEG and is not generally recommended in $\mathrm{RIG}^{45}$ which may have resulted in a reduction in the rates of peristomal infection and other acute infectious complications in PEG; ${ }^{8,9,31,33}$ second, the tubes used in the two methods differ in shape, as the RIG tubes are relatively thinner in diameter (10-14 F) than the PEG tubes (20 F), and clogging of the feeding tube or tube blockage would be more frequent in tubes of smaller diameters; ${ }^{1}$ furthermore, as RIG tubes are fixed with a balloonretention system, they are not as securely fixed as PEG tubes, and peristomal leakage or tube displacement would be more frequent. ${ }^{28,33}$ These differences in tubes may have induced a significant increase in the rate of tube related complications. ${ }^{9}$ Furthermore, there were differences in underlying diseases between the two groups. The PEG group contained more patients with neurological impairment, while the RIG group included more patients with head and neck cancer. These differences might have influenced the outcomes of the study.

In a subgroup analysis of patients with motor neuron disease, the 30-day mortality rates of the PEG and RIG groups were not significantly different. The PEG procedure in patients with motor neuron disease was generally considered more risky due to probable respiratory distress. ${ }^{24}$ However, our results provide evidence that PEG is not inferior to RIG in these patients.

The subgroup analysis of patients with head and neck cancer demonstrated a statistically significant reduction in the 30-day mortality rate of the PEG group compared to that of RIG group. In general, a radiological approach is preferred in patients with head and neck cancer because of concerns about tumor seeding and technical problems with oral insertion due to the location of the tumor. However, only three studies were conducted in patients with head and neck cancer to compare 30-day mortality in PEG versus RIG; the small study effect demonstrated by the Egger's test was not significant $(P=0.06)$. Considering these results, $P E G$ is thought to be a safe method for gastrostomy even in patients with head and neck cancer.

There are several limitations to this study. First, most studies enrolled in this meta-analysis were observational studies without randomization of insertion methods, and all the groups were not matched exactly. Although gastrostomy has been widely used, there is a lack of randomized trials on this issue. Because of difficult ethical problems surrounding feeding and the issues of vulnerability in patients with impaired neurology, large randomized controlled studies are not easily performed and are less motivating in changing clinical practice. ${ }^{46}$ Although many researchers argue that combining analyses of RCTs and NRCTs in a meta-analysis should be avoided, both study designs contain strengths and weaknesses in principle. Additionally, including results from observational studies has been suggested as a means of improving the inferences made only by RCTs. We searched peer-reviewed articles only, and excluded biased studies. Therefore, we believe that this meta-analysis including RCTs and NRCTs had the power to provide better inferences on an issue rarely examined in the context of a RCT. Second, we could not collect data on albumin and CRP levels, which are known as predictive factors for 30-day mortality after gastrostomy. ${ }^{1}$ Furthermore, information on the use of prophylactic antibiotics could not be collected, as these could have introduced selection bias. However, we enrolled a comparative study with a higher quality to minimize these uninvestigated selection biases.

In conclusion, PEG was associated with a lower probability of 30-day mortality, suggesting that it could become the first choice for long-term enteral tube feeding. Further randomized prospective studies are needed to evaluate and compare the safety of gastrostomy tube insertion by the two different methods investigated in this study.

\section{ACKNOWLEDGEMENT}

Seoul National University Hospital Medical Research Col- 
laborating Center (SNUH MRCC) contributed to the statistical analysis in this report.

\section{REFERENCES}

1. Lee C, Im JP, Kim JW, et al. Risk factors for complications and mortality of percutaneous endoscopic gastrostomy: a multicenter, retrospective study. Surg Endosc 2013;27:3806-3815.

2. Gauderer MW, Ponsky JL, Izant RJ Jr. Gastrostomy without laparotomy: a percutaneous endoscopic technique. J Pediatr Surg 1980;15:872-875.

3. Preshaw RM. A percutaneous method for inserting a feeding gastrostomy tube. Surg Gynecol Obstet 1981;152:658-660.

4. Sanders DS, Carter MJ, D'Silva J, James G, Bolton RP, Bardhan KD. Survival analysis in percutaneous endoscopic gastrostomy feeding: a worse outcome in patients with dementia. Am J Gastroenterol 2000;95:1472-1475.

5. Richter-Schrag HJ, Richter S, Ruthmann O, Olschewski M, Hopt UT, Fischer A. Risk factors and complications following percutaneous endoscopic gastrostomy: a case series of 1041 patients. Can J Gastroenterol 2011;25:201-206.

6. Zopf Y, Maiss J, Konturek P, Rabe C, Hahn EG, Schwab D. Predictive factors of mortality after PEG insertion: guidance for clinical practice. JPEN J Parenter Enteral Nutr 2011;35:50-55.

7. Lowe AS, Laasch HU, Stephenson S, et al. Multicentre survey of radiologically inserted gastrostomy feeding tube (RIG) in the UK. Clin Radiol 2012;67:843-854.

8. Laskaratos FM, Walker M, Walker M, et al. Predictive factors for early mortality after percutaneous endoscopic and radiologically-inserted gastrostomy. Dig Dis Sci 2013;58:3558-3565.

9. ProGas Study Group. Gastrostomy in patients with amyotrophic lateral sclerosis (ProGas): a prospective cohort study. Lancet Neurol 2015;14:702-709.

10. Park WY, Lee TH, Lee JS, et al. Reappraisal of pneumoperitoneum after percutaneous endoscopic gastrostomy. Intest Res 2015;13:313-317.

11. Janes SE, Price CS, Khan S. Percutaneous endoscopic gastrostomy: 30-day mortality trends and risk factors. J Postgrad Med 2005;51:23-28.

12. Blomberg J, Lagergren P, Martin L, Mattsson F, Lagergren J. Albumin and C-reactive protein levels predict short-term mortality after percutaneous endoscopic gastrostomy in a prospective cohort study. Gastrointest Endosc 2011;73:29-36.

13. Moher D, Liberati A, Tetzlaff J, Altman DG; PRISMA Group. Preferred reporting items for systematic reviews and metaanalyses: the PRISMA statement. PLoS Med 2009;6:e1000097. doi: 10.1371/journal.pmed.1000097.
14. Jadad AR, Moore RA, Carroll D, et al. Assessing the quality of reports of randomized clinical trials: is blinding necessary? Control Clin Trials 1996;17:1-12.

15. Slim K, Nini E, Forestier D, Kwiatkowski F, Panis Y, Chipponi J. Methodological index for non-randomized studies (minors): development and validation of a new instrument. ANZ J Surg 2003;73:712-716.

16. Lewis S, Jackson S, Latchford A. Randomized study of radiologic vs endoscopic placement of gastrojejunostomies in patients at risk of aspiration pneumonia. Nutr Clin Pract 2014;29:498-503.

17. Nugent B, Lewis S, O'Sullivan JM. Enteral feeding methods for nutritional management in patients with head and neck cancers being treated with radiotherapy and/or chemotherapy. Cochrane Database Syst Rev 2013;(1):CD007904. doi: 10.1002/14651858.CD007904.pub3.

18. McAllister P, MacIver C, Wales C, et al. Gastrostomy insertion in head and neck cancer patients: a 3 year review of insertion method and complication rates. Br J Oral Maxillofac Surg 2013;51:714-718.

19. Allen JA, Chen R, Ajroud-Driss S, et al. Gastrostomy tube placement by endoscopy versus radiologic methods in patients with ALS: a retrospective study of complications and outcome. Amyotroph Lateral Scler Frontotemporal Degener 2013;14:308-314.

20. La Nauze RJ, Collins K, Lyon S, et al. Outcomes of percutaneous endoscopic gastrostomy versus radiologically inserted gastrostomy tube insertion at a tertiary hospital. e-SPEN Journal 2012;7:e144-e148. doi:10.1016/j.clnme.2012.05.001.

21. Burkitt P, Carter LM, Smith AB, Kanatas A. Outcomes of percutaneous endoscopic gastrostomy and radiologically inserted gastrostomy in patients with head and neck cancer: a systematic review. Br J Oral Maxillofac Surg 2011;49:516-520.

22. Rio A, Ellis C, Shaw C, et al. Nutritional factors associated with survival following enteral tube feeding in patients with motor neurone disease. J Hum Nutr Diet 2010;23:408-415.

23. Leeds JS, McAlindon ME, Grant J, Robson HE, Lee FK, Sanders DS. Survival analysis after gastrostomy: a single-centre, observational study comparing radiological and endoscopic insertion. Eur J Gastroenterol Hepatol 2010;22:591-596.

24. Blondet A, Lebigot J, Nicolas G, et al. Radiologic versus endoscopic placement of percutaneous gastrostomy in amyotrophic lateral sclerosis: multivariate analysis of tolerance, efficacy, and survival. J Vasc Interv Radiol 2010;21:527-533.

25. Grant DG, Bradley PT, Pothier DD, et al. Complications following gastrostomy tube insertion in patients with head and neck cancer: a prospective multi-institution study, systematic review and meta-analysis. Clin Otolaryngol 2009;34:103-112. 
26. Galaski A, Peng WW, Ellis M, Darling P, Common A, Tucker E. Gastrostomy tube placement by radiological versus endoscopic methods in an acute care setting: a retrospective review of frequency, indications, complications and outcomes. Can J Gastroenterol 2009;23:109-114.

27. MacLean AA, Alvarez NR, Davies JD, Lopez PP, Pizano LR. Complications of percutaneous endoscopic and fluoroscopic gastrostomy tube insertion procedures in 378 patients. Gastroenterol Nurs 2007;30:337-341.

28. Eze N, Jefford JM, Wolf D, Williamson P, Neild P. PEG and RIG tube feeding in head and neck patients: a retrospective review of complications and outcome. J Eval Clin Pract 2007;13:817-819.

29. Shaw AS, Ampong MA, Rio A, et al. Survival of patients with ALS following institution of enteral feeding is related to preprocedure oximetry: a retrospective review of 98 patients in a single centre. Amyotroph Lateral Scler 2006;7:16-21.

30. Rustom IK, Jebreel A, Tayyab M, England RJ, Stafford ND. Percutaneous endoscopic, radiological and surgical gastrostomy tubes: a comparison study in head and neck cancer patients. J Laryngol Otol 2006;120:463-466.

31. Silas AM, Pearce LF, Lestina LS, et al. Percutaneous radiologic gastrostomy versus percutaneous endoscopic gastrostomy: a comparison of indications, complications and outcomes in 370 patients. Eur J Radiol 2005;56:84-90.

32. Desport JC, Mabrouk T, Bouillet P, Perna A, Preux PM, Couratier P. Complications and survival following radiologically and endoscopically-guided gastrostomy in patients with amyotrophic lateral sclerosis. Amyotroph Lateral Scler Other Motor Neuron Disord 2005;6:88-93.

33. Neeff M, Crowder VL, McIvor NP, Chaplin JM, Morton RP. Comparison of the use of endoscopic and radiologic gastrostomy in a single head and neck cancer unit. ANZ J Surg 2003;73:590593.

34. Laasch HU, Wilbraham L, Bullen K, et al. Gastrostomy insertion: comparing the options: PEG, RIG or PIG? Clin Radiol 2003;58:398-405.

35. Thornton FJ, Fotheringham T, Alexander M, Hardiman O, McGrath FP, Lee MJ. Amyotrophic lateral sclerosis: enteral nutrition provision: endoscopic or radiologic gastrostomy? Radiology 2002;224:713-717.
36. Kohout P, Manák J, Vacek Z, Tucek D. Puncture gastrostomy in oncologic patients. Vnitr Lek 2002;48:626-628.

37. Dinkel HP, Beer KT, Zbären P, Triller J. Establishing radiological percutaneous gastrostomy with balloon-retained tubes as an alternative to endoscopic and surgical gastrostomy in patients with tumours of the head and neck or oesophagus. Br J Radiol 2002;75:371-377.

38. Bazarah SM, Al-Rawas M, Akbar H, Qari Y. Percutaneous gastrostomy and gastrojejunostomy: radiological and endoscopic approach. Ann Saudi Med 2002;22:38-42.

39. Hoffer EK, Cosgrove JM, Levin DQ, Herskowitz MM, Sclafani SJ. Radiologic gastrojejunostomy and percutaneous endoscopic gastrostomy: a prospective, randomized comparison. J Vasc Interv Radiol 1999;10:413-420.

40. Cosentini EP, Sautner T, Gnant M, Winkelbauer F, Teleky B, Jakesz R. Outcomes of surgical, percutaneous endoscopic, and percutaneous radiologic gastrostomies. Arch Surg 1998;133:1076-1083.

41. Barkmeier JM, Trerotola SO, Wiebke EA, et al. Percutaneous radiologic, surgical endoscopic, and percutaneous endoscopic gastrostomy/gastrojejunostomy: comparative study and cost analysis. Cardiovasc Intervent Radiol 1998;21:324-328.

42. Wollman B, D’Agostino HB. Percutaneous radiologic and endoscopic gastrostomy: a 3-year institutional analysis of procedure performance. AJR Am J Roentgenol 1997;169:1551-1553.

43. Wollman B, D’agostino HB, Walus-Wigle JR, Easter DW, Beale A. Percutaneous radiologic and endoscopic gastrostomy: a two-year evaluation. Minim Invasive Ther Allied Technol 1996;5:544-546.

44. Wollman B, D'Agostino HB, Walus-Wigle JR, Easter DW, Beale A. Radiologic, endoscopic, and surgical gastrostomy: an institutional evaluation and meta-analysis of the literature. Radiology 1995;197:699-704.

45. ASGE Standards of Practice Committee, Jain R, Maple JT, et al. The role of endoscopy in enteral feeding. Gastrointest Endosc 2011;74:7-12.

46. Kelly AG, Sahin B, Holloway RG. Ethical considerations in stroke patients. Curr Opin Neurol 2014;27:61-65. 